\title{
Juvenile fish assemblages in the Jinju Bay region, Korea
}

\author{
Se Hun Myoung ${ }^{1}$, Seok Nam Kwak², Jin-Koo Kim³ ${ }^{3 *}$, Won-Chan Lee ${ }^{4}$, Jeong Bae Kim ${ }^{4}$ Hyung Chul Kim ${ }^{4}$ and \\ Jane E. Williamson'
}

\begin{abstract}
Assemblages of juvenile fish and associated abiotic parameters were investigated inside and outside Jinju Bay in southern Korea, on a monthly basis from December 2014 to November 2015. Fluctuations in water temperature and salinity were larger inside than outside the bay. In total, 534,657 individuals per square kilometre from 81 fish species and 47 families were collected during the study period. The most dominant species was Nuchequula nuchalis both inside (25.6\%) and outside (26.9\%) the bay. The next dominant species were Thryssa kammalensis (17.9\%) and Zoarces gillii (16.0\%) inside the bay and Liparis tanakae (16.9\%) and T. kammalensis (9.0\%) outside the bay. Forty species (33\% of total number of individuals) of young fish were recorded inside the bay and 47 species (52\%) outside the bay. Therefore, it appears that a diversity of fish use nursery grounds inside and outside Jinju Bay. In particular, the following six species appeared: Z. gillii, Pleuronichthys cornutus, L. tanakae, Hemitripterus villosus, Pennahia argentata, and Xenocephalus elongates. Due to assemblage differences for fishes within Jinju Bay and outside the bay, management of both areas is required to maintain current diversity of species in the region.
\end{abstract}

Keywords: Bay ecology, Young fish, Teleost, Nursery, Jinju Bay, Nuchequula nuchalis, Thryssa kammalensis, Liparis tanakae

\section{Introduction}

Coastal bay environments are highly variable, particularly in terms of water temperature, salinity, oxygen, sea level, nutrient availability, and turbidity. These variabilities often create unfavourable conditions for marine organisms within bay ecosystems (Faria et al. 2006). Such variabilities, however, can also provide favourable conditions for early stages of fishes during ontogenesis, such as increased availability of nutrients from terrestrial discharge that can result in an abundant food supply (Selleslagh et al. 2009; Newton et al. 2014; Álvarez et al. 2015). Bays can also offer shelter and protection from predators for larval and juvenile stages of fishes (Allen 1982; Able and Fahay 2010; Song et al. 2012), as well as facilitating larval movement via protection from wave action (Swearer et al. 1999). As increases in shelter and food supply are generally positively

\footnotetext{
* Correspondence: taengko@hanmail.net

${ }^{3}$ Department of Marine Biology, Pukyong National University, Busan 48513, South Korea

Full list of author information is available at the end of the article
}

correlated with rapid growth and high survival rates in the early stages of fishes, bays are generally considered as important spawning and nursery grounds worldwide (Vasconcelos et al. 2010; Grol et al. 2011; Newton et al. 2014; Lin et al. 2016). Many larvae and young fish (Has species characteristics, but sexually immature) inhabit these regions. Understanding the composition of the assemblages in these areas directs appropriate management of the species concerned.

Jinju Bay is located in the middle southern coast of Korea and is surrounded by Sacheon, Hadong, and Namhae provinces. The bay is semi-enclosed and highly influenced by the Nam River Dam, $9.5 \mathrm{~km}$ north from the tip of the estuary that feeds into the bay. During the monsoonal season in Korea (July to September), increases in freshwater significantly impact the associated coastal marine ecosystems (Yeo and Park 1997; Park 2005). The bay is also considered a spawning and nursery ground for various marine organisms including commercial and recreational species (Kurita et al. 2017;

(c) The Author(s). 2020 Open Access This article is licensed under a Creative Commons Attribution 4.0 International License, which permits use, sharing, adaptation, distribution and reproduction in any medium or format, as long as you give appropriate credit to the original author(s) and the source, provide a link to the Creative Commons licence, and indicate if changes were made. The images or other third party material in this article are included in the article's Creative Commons licence, unless indicated otherwise in a credit line to the material. If material is not included in the article's Creative Commons licence and your intended use is not permitted by statutory regulation or exceeds the permitted use, you will need to obtain permission directly from the copyright holder. To view a copy of this licence, visit http://creativecommons.org/licenses/by/4.0/. 
Yamane et al. 2019), and has long been used for shellfish farming because of its protection against wave action from the open sea. Approximately one quarter of the bay is comprised of intertidal habitat, which potentially accumulates organic pollutants derived from urban and human activities.

The influence of freshwater discharge from the Nam River Dam on the coastal environment and associated biological communities has previously been reported. Such impacts include stratification and destratification processes, which change the availability of nutrients and temperature gradients in the water column (Jung and Ro 2010; Kang et al. 2011), and circulation flows around the bay (Kim et al. 2010), all of which are documented to affect the distribution of phytoplankton (Oh et al. 2007) and polychaete (Kang et al. 2002) communities. Impacts of this freshwater discharge on the structure of fish populations have not been assessed.

This research investigates monthly changes in the species composition and community structure of juvenile fishes in Jinju Bay and just outside the bay in relation to the salinity gradient caused by the Nam River Dam. Such studies are pivotal in understanding the ecological function and optimizing sustainable management plans for the Jinju Bay area.

\section{Materials and methods}

\section{Sampling and environmental observations}

Samples were collected at two stations in waters inside and outside Jinju Bay, approximately $15 \mathrm{~km}$ apart (Fig. 1). Jinju Bay is shallow and heavily affected by coastal waters, while outside the bay the waters are deeper and less affected by coastal waters and freshwater flows from the Nam River Dam. The two sampling stations were classified according to adjacent geographic features and their distance from the Nam River (Kim et al. 2010; Kang et al. 2011). The inside station was located at the lower reaches of Nam River, surrounded by villages, inlands, islands, and reefs, while the outside station was exposed to open ocean from the southeastern inlet (Fig. 1).

Fish samples were collected using a small beam trawl (beam length $6 \mathrm{~m}$; vertical net opening $1 \mathrm{~m}$; mesh size $20 \mathrm{~mm}$ ) monthly from December 2014 to November 2015 at both stations, with the exception of February 2015, where collection was not possible. The net was towed at a speed of 1.6-1.8 knots for 60 min (daytime). Immediately after capture, fish samples were snap frozen to $-20{ }^{\circ} \mathrm{C}$ then taken to the laboratory at Pukyong National University. Once at the laboratory, the total length (TL) of each fish was measured to the nearest millimetre. Bottom water temperature and salinity measurements were also taken monthly using a conductivitytemperature-depth (CTD) metre (SBE-19 plus, Sea-Bird Electronics, Inc.). The CTD metre was also used to measure the depth of the two stations.

\section{Data analysis}

All fish species were identified to the lowest possible taxonomic classification according to Kim et al. (2005) and Nakabo (2013). The scientific names and taxonomic classifications of fishes followed Nelson et al. (2016) and Kim and Ryu (2016). And the species collected according to Elliott and Dewailly (1995) were classified into six types according to purpose: estuarine residents (ER),

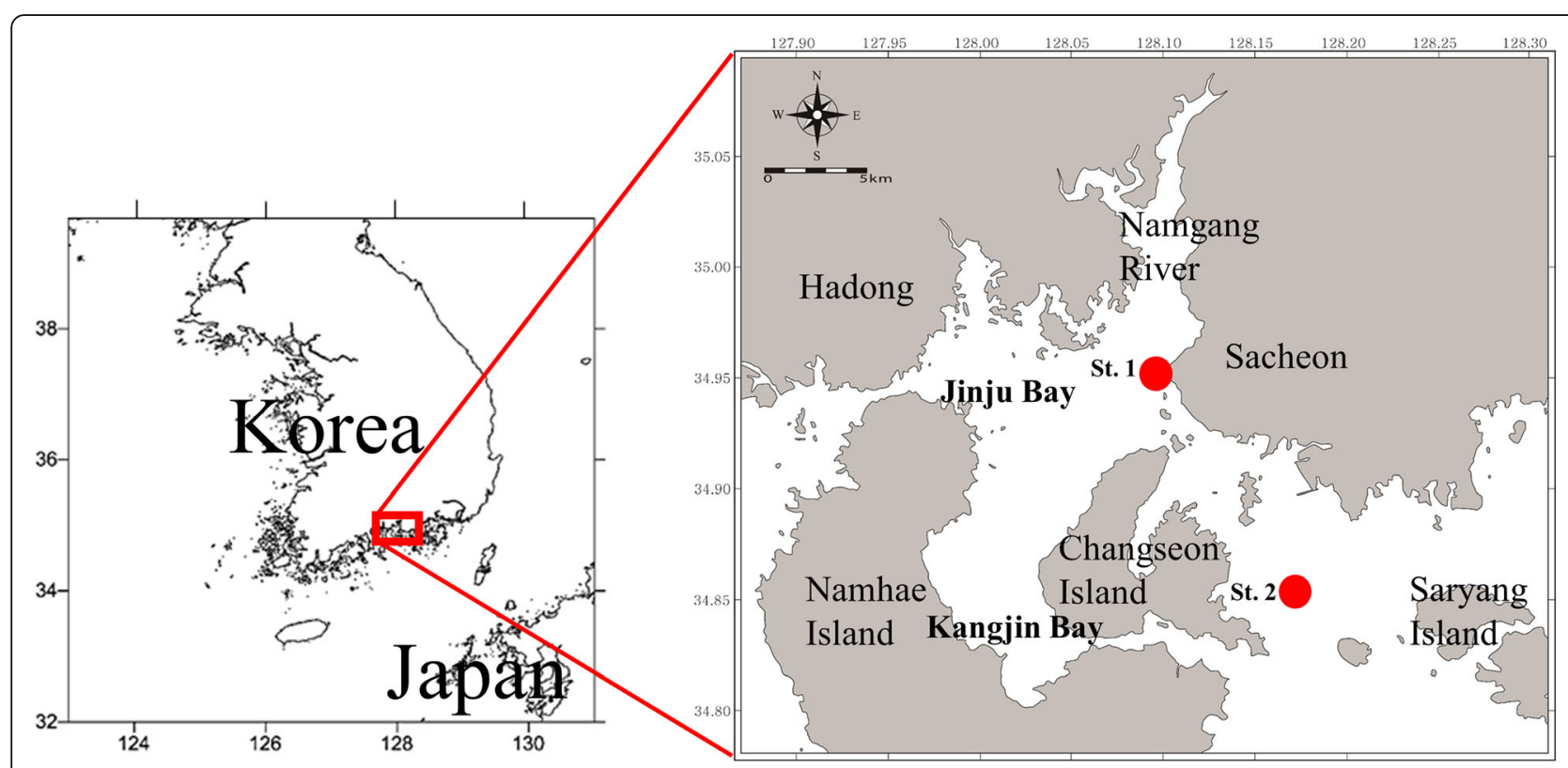

Fig. 1 Location of the stations inside and outside of Jinju Bay, Korea 
marine adventitious visitors (MA), diadromous (catadromous/anadromous) migrants (CA), and marine seasonal. migrants (MS), marine juvenile migrants (nursery species) (MJ), or freshwater adventitious visitors (FW). The total length and wet body weight of each individual was measured to the nearest millimetre and gram, respectively. The abundance of each species was obtained using the swept area method (number of individuals per $\mathrm{km}^{2}$ ). Species occurring more than six times (over 50\%) during the survey period were considered resident species.

A one-way ANOVA followed by a post hoc Bonferroni's test, with sample site and season as fixed factors, was used to analyse the abundance data. All species were considered in the analyses, and abundances were $\log (x+1)$ transformed. For the eight most numerically abundant species (comprising greater than $6.0 \%$ of the total population), variations in seasonal [spring (March-May), summer (June-August), autumn (September-November), and winter (December-February)] mean abundance were analysed.

A Mann-Whitney $U$ test was used to examine differences in the number of fish species collected from inside and outside Jinju Bay. The community-level variable of fish assemblage was expressed as a species diversity index $\left(\mathrm{H}^{\prime}\right.$, Shannon and Weaver 1949) using the number of species and its abundance data. A Bray-Curtis similarity matrix was constructed based on the abundance of the fish species (Bray and Curtis 1957). Before calculation, a logarithmic transformation $\left[\log _{10}(x+1)\right]$ was applied to the data to decrease the effects of a few but extremely abundant species. Cluster analysis was carried out using the Bray-Curtis similarity. A similarity percentage (SIMPER) was then used to examine which species contributed most to the differences among samples. A non-metric multidimensional scaling (nMDS) ordination was further visualized to examine the cluster relationship on a two-dimensional plot. All multivariate analyses were performed using PRIMER statistical package version 6.0 (Clarke and Gorley 2006).

In addition, relationships between fish abundance and environmental factors (i.e. water temperature, salinity, depth, water transparency) were analyzed using canonical correspondence analysis (CCA). The relative contributions of environmental variables to the observed differences were assessed using correlation coefficients for relationships
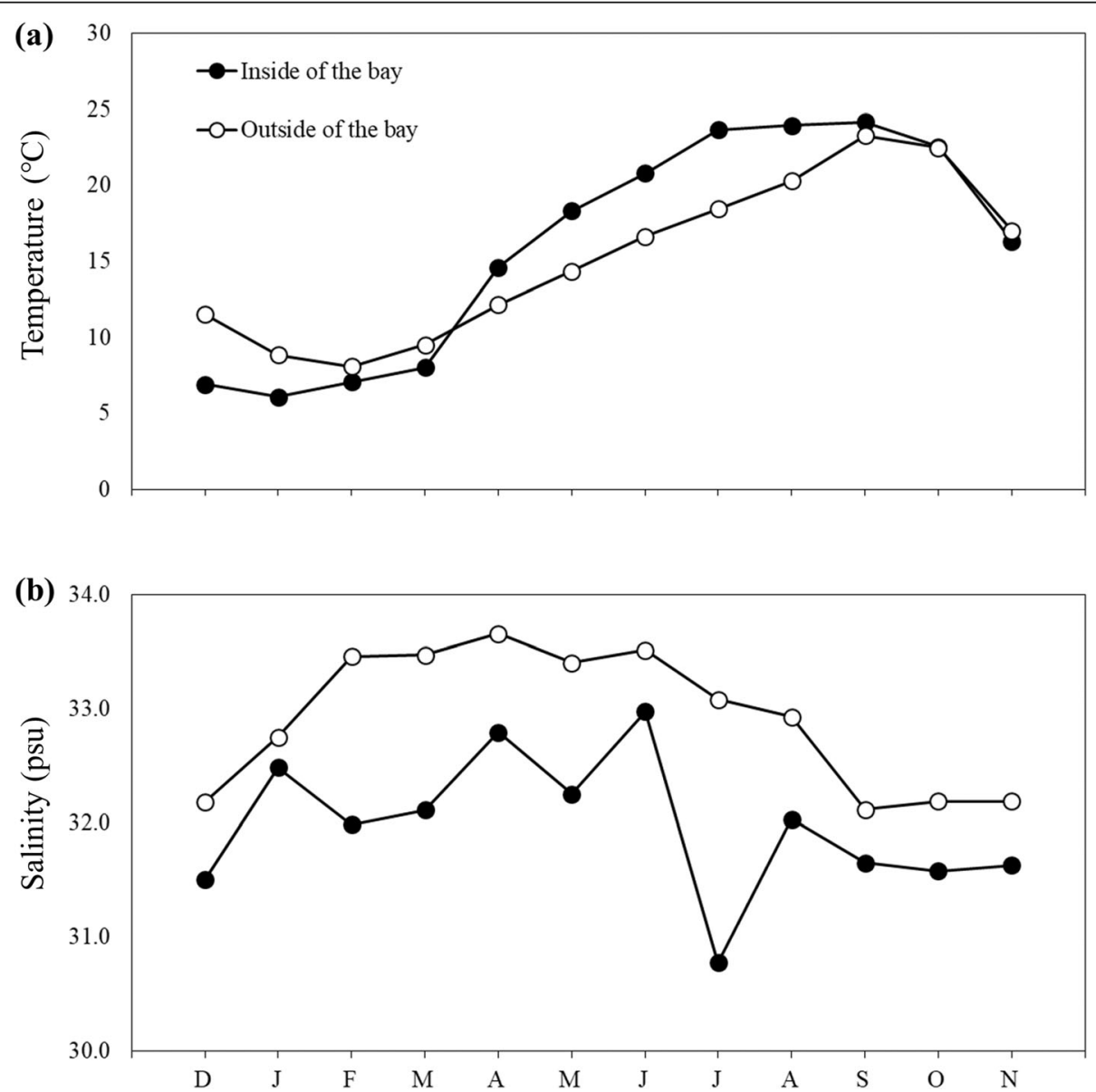

Fig. 2 Monthly variations in the bottom water temperature (a) and salinity (b) from inside (black circle) and outside (open circle) of Jinju Bay 
between each fish assemblage of common fish species and the canonical axis. To avoid overestimation caused by less frequently occurring species, only those accounting for over $0.5 \%$ of the total percentage of abundance (resident fish) were used for this analysis. The test was performed using the package Excel XLSTAT V.7.5.2 (Add-in-software, http://www.xlstat.com). Data were log transformed $\log (x+1)$ prior to analysis.

\section{Results}

Environmental variables

Depth between the stations differed, with depth inside Jinju Bay measured at $10.6 \mathrm{~m}$ and the outside at $18.6 \mathrm{~m}$. Bottom water temperatures showed similar trends between the two stations, with a lower temperature measured during winter than during summer (Fig. 2). The extent of seasonal fluctuations in water temperature were greater at the station inside

Table 1 List of fish species and mean abundance of species (per $\mathrm{km}^{2}$ ) in inside and outside of Jinju Bay, Korea, from December 2014 to November 2015

\begin{tabular}{|c|c|c|c|c|c|c|}
\hline \multirow[t]{2}{*}{ Family } & \multirow[t]{2}{*}{ Species } & \multirow[t]{2}{*}{$E R^{a}$} & \multicolumn{2}{|c|}{ abundance } & \multicolumn{2}{|c|}{ body length } \\
\hline & & & inside & outside & inside & outside \\
\hline Rajidae & Okamejei kenojei & MA & 250 & 3200 & $9.8-31.3$ & $9.2-37.6$ \\
\hline Dasyatidae & Dasyatis akajei & MA & 100 & & $48.9-49$ & \\
\hline Muraenesocidae & Muraenesox cinereus & MA & 550 & 1000 & $34.1-53.8$ & 29.6-51.4 \\
\hline Congridae & Conger myriaster & ER & 2200 & 2950 & $22.5-54.5$ & $11.5-41.3$ \\
\hline \multirow[t]{2}{*}{ Engraulidae } & Thryssa kammalensis & MJ & 33797 & 31297 & $7.0-13.5$ & 4.4-13.3 \\
\hline & Thryssa adelae & MA & 50 & 800 & 14.1 & $9.0-14.3$ \\
\hline \multirow[t]{2}{*}{ Clupeidae } & Sardinella zunasi & MJ & 50 & 50 & 12.1 & $9.4-9.4$ \\
\hline & Konosirus punctatus & MJ & & 100 & & $11.2-12.0$ \\
\hline Synodontidae & Saurida microlepis & MA & 100 & 300 & $27.6-41.7$ & 7.6-39.4 \\
\hline Macrouridae & Coelorinchus multispinulosus & MA & & 100 & & 18.2-19.4 \\
\hline Gadidae & Gadus macrocephalus & MJ & 100 & & $7.1-70.0$ & \\
\hline Lophiidae & Lophius litulon & MA & 50 & 100 & 32.1 & $6.1-12.3$ \\
\hline Syngnathidae & Hippocampus mohnikei & ER & 100 & & $6.0-7.1$ & \\
\hline \multirow[t]{6}{*}{ Scorpaenidae } & Sebastes inermis & MJ & 50 & & 7.4 & \\
\hline & Scorpaena neglecta & MJ & 50 & & 3.1 & \\
\hline & Inimicus japonicus & MJ & 2000 & 1450 & $5.2-22.9$ & $5.4-29.5$ \\
\hline & Scorpaena onaria & MA & 100 & & $5.3-6.0$ & \\
\hline & Sebastiscus marmoratus & MJ & & 50 & & $19.5-19.5$ \\
\hline & Minous monodactylus & MA & & 50 & & $12.4-12.4$ \\
\hline \multirow[t]{2}{*}{ Aploactinidae } & Hypodytes rubripinnis & MJ & 500 & 1450 & $4.2-7.4$ & $3.8-8.0$ \\
\hline & Erisphex pottii & MA & 150 & & $7.3-8.9$ & \\
\hline Triglidae & Chelidonichthys spinosus & MA & 450 & 2400 & 16.4-30.6 & $15.5-26.0$ \\
\hline \multirow[t]{2}{*}{ Platycephalidae } & Cociella crocodila & MJ & 200 & & $9.5-18.5$ & \\
\hline & Platycephalus indicus & MJ & 1200 & 2400 & $6.4-29.5$ & $11.4-41.0$ \\
\hline \multirow[t]{2}{*}{ Hexagrammidae } & Hexagrammos agrammus & MA & 50 & & 13.8 & \\
\hline & Hexagrammos otakii & MJ & 500 & 50 & $9.9-24.7$ & 21.4 \\
\hline \multirow[t]{3}{*}{ Cottidae } & Pseudoblennius cottoides & MJ & 350 & & $4.6-5.5$ & \\
\hline & Alcichthys elongatus & MJ & 350 & & & \\
\hline & Ricuzenius pinetorum & MJ & 7649 & 12099 & $4.3-8.5$ & $5.5-8.8$ \\
\hline Hemitripteridae & Hemitripterus villosus & MJ & 1700 & 1850 & $2.2-9.3$ & $4.1-28.4$ \\
\hline Liparidae & Liparis tanakae & MJ & 5900 & 58445 & 4.3-55.4 & $5.2-52.6$ \\
\hline Moronidae & Lateolabrax maculatus & MJ & & 50 & & 20.9 \\
\hline Acropomatidae & Malakichthys wakiyae & MA & & 50 & & 4.3 \\
\hline Apogonidae & Apogon lineatus & MA & 1750 & 6799 & $5.6-9.0$ & $3.4-9.7$ \\
\hline
\end{tabular}

${ }^{\mathrm{a} E c o l o g i c a l}$ guilds: $E R$ estuarine residents, $M A$ marine adventitious visitors, $M J$ marine juvenile migrants (nursery species) 
Table 2 Species composition of fishes collected by shrimp beam trawl inside of Jinju Bay, Korea, from December 2014 to November 2015 (unit: ind. $/ \mathrm{km}^{2}$ )

\begin{tabular}{|c|c|c|c|c|c|c|c|c|c|c|c|c|c|}
\hline Scientific name & Dec. & Jan. & Mar. & Apr. & May & Jun. & Jul. & Aug. & Sep. & Oct. & Nov. & Total & $\%$ \\
\hline Okamejei kenojei & 0 & 0 & 50 & 50 & 50 & 0 & 50 & 50 & 0 & 0 & 0 & 250 & 0.1 \\
\hline Dasyatis akajei & 0 & 0 & 0 & 0 & 50 & 0 & 0 & 0 & 0 & 50 & 0 & 100 & 0.1 \\
\hline Muraenesox cinereus & 0 & 0 & 0 & 0 & 0 & 0 & 0 & 450 & 100 & 0 & 0 & 550 & 0.3 \\
\hline Conger myriaster & 250 & 300 & 0 & 0 & 350 & 250 & 350 & 300 & 0 & 150 & 250 & 2,200 & 1.2 \\
\hline Thryssa kammalensis & 0 & 0 & 0 & 0 & 0 & 0 & 6,100 & 27,548 & 100 & 0 & 50 & 33,797 & 17.9 \\
\hline Thryssa adelae & 0 & 0 & 0 & 0 & 0 & 0 & 0 & 50 & 0 & 0 & 0 & 50 & + \\
\hline Sardinella zunasi & 0 & 0 & 0 & 0 & 0 & 0 & 50 & 0 & 0 & 0 & 0 & 50 & + \\
\hline Saurida microlepis & 0 & 0 & 0 & 0 & 0 & 0 & 50 & 0 & 0 & 0 & 50 & 100 & 0.1 \\
\hline Gadus macrocephalus & 50 & 0 & 0 & 0 & 50 & 0 & 0 & 0 & 0 & 0 & 0 & 100 & 0.1 \\
\hline Lophius litulon & 0 & 0 & 0 & 50 & 0 & 0 & 0 & 0 & 0 & 0 & 0 & 50 & + \\
\hline Hippocampus mohnikei & 0 & 0 & 0 & 0 & 0 & 100 & 0 & 0 & 0 & 0 & 0 & 100 & 0.1 \\
\hline Sebastes inermis & 50 & 0 & 0 & 0 & 0 & 0 & 0 & 0 & 0 & 0 & 0 & 50 & + \\
\hline Scorpaena neglecta & 0 & 0 & 50 & 0 & 0 & 0 & 0 & 0 & 0 & 0 & 0 & 50 & + \\
\hline Inimicus japonicus & 0 & 0 & 0 & 150 & 550 & 1,000 & 50 & 50 & 200 & 0 & 0 & 2,000 & 1.1 \\
\hline Scorpaena onaria & 0 & 0 & 0 & 0 & 0 & 100 & 0 & 0 & 0 & 0 & 0 & 100 & 0.1 \\
\hline Hypodytes rubripinnis & 350 & 100 & 0 & 50 & 0 & 0 & 0 & 0 & 0 & 0 & 0 & 500 & 0.3 \\
\hline Erisphex pottii & 150 & 0 & 0 & 0 & 0 & 0 & 0 & 0 & 0 & 0 & 0 & 150 & 0.1 \\
\hline Chelidonichthys spinosus & 100 & 0 & 0 & 0 & 100 & 100 & 0 & 0 & 100 & 0 & 50 & 450 & 0.2 \\
\hline Cociella crocodila & 100 & 0 & 0 & 0 & 0 & 100 & 0 & 0 & 0 & 0 & 0 & 200 & 0.1 \\
\hline Platycephalus indicus & 700 & 100 & 0 & 200 & 50 & 0 & 0 & 50 & 0 & 0 & 100 & 1,200 & 0.6 \\
\hline Hexagrammos agrammus & 50 & 0 & 0 & 0 & 0 & 0 & 0 & 0 & 0 & 0 & 0 & 50 & + \\
\hline Hexagrammos otakii & 250 & 50 & 50 & 50 & 100 & 0 & 0 & 0 & 0 & 0 & 0 & 500 & 0.3 \\
\hline Pseudoblennius cottoides & 0 & 0 & 0 & 0 & 350 & 0 & 0 & 0 & 0 & 0 & 0 & 350 & 0.2 \\
\hline Cottidae sp.1 & 0 & 0 & 0 & 0 & 0 & 350 & 0 & 0 & 0 & 0 & 0 & 350 & 0.2 \\
\hline Ricuzenius pinetorum & 5,400 & 1,350 & 750 & 150 & 0 & 0 & 0 & 0 & 0 & 0 & 0 & 7,649 & 4.1 \\
\hline Hemitripterus villosus & 0 & 0 & 1,200 & 450 & 50 & 0 & 0 & 0 & 0 & 0 & 0 & 1,700 & 0.9 \\
\hline Liparis tanakae & 50 & 100 & 2,350 & 3,300 & 100 & 0 & 0 & 0 & 0 & 0 & 0 & 5,900 & 3.1 \\
\hline Apogon lineatus & 0 & 0 & 0 & 0 & 100 & 1,500 & 100 & 0 & 50 & 0 & 0 & 1,750 & 0.9 \\
\hline Sillago japonica & 0 & 0 & 0 & 0 & 850 & 200 & 600 & 1,650 & 250 & 200 & 200 & 3,950 & 2.1 \\
\hline Trachurus japonicus & 0 & 0 & 0 & 0 & 50 & 100 & 0 & 0 & 0 & 0 & 0 & 150 & 0.1 \\
\hline Nuchequula nuchalis & 450 & 0 & 300 & 150 & 600 & 50 & 4,450 & 36,297 & 300 & 3,450 & 2,250 & 48,296 & 25.6 \\
\hline Acanthopagrus schlegelii & 100 & 100 & 0 & 0 & 0 & 0 & 0 & 0 & 0 & 0 & 0 & 200 & 0.1 \\
\hline Johnius grypotus & 0 & 0 & 0 & 0 & 0 & 0 & 0 & 250 & 0 & 0 & 0 & 250 & 0.1 \\
\hline Pennahia argentata & 200 & 150 & 0 & 0 & 150 & 0 & 0 & 450 & 1,250 & 600 & 200 & 3,000 & 1.6 \\
\hline Upeneus japonicus & 0 & 0 & 0 & 0 & 0 & 0 & 0 & 0 & 50 & 50 & 100 & 200 & 0.1 \\
\hline Ditrema temminckii temminckii & 50 & 0 & 0 & 0 & 0 & 0 & 0 & 0 & 0 & 0 & 0 & 50 & + \\
\hline Zoarces gillii & 250 & 700 & 50 & 16,549 & 11,749 & 750 & 0 & 0 & 0 & 50 & 0 & 30,098 & 16.0 \\
\hline Chirolophis wui & 0 & 0 & 50 & 50 & 0 & 0 & 0 & 0 & 0 & 0 & 0 & 100 & 0.1 \\
\hline Pholis nebulosa & 750 & 400 & 450 & 50 & 150 & 50 & 50 & 0 & 0 & 100 & 0 & 2,000 & 1.1 \\
\hline Pholis fangi & 700 & 1,250 & 1,150 & 6,150 & 2,900 & 150 & 0 & 0 & 0 & 0 & 200 & 12,499 & 6.6 \\
\hline Champsodon snyderi & 0 & 0 & 0 & 0 & 0 & 50 & 50 & 0 & 0 & 0 & 0 & 100 & 0.1 \\
\hline Parapercis sexfasciata & 250 & 150 & 100 & 150 & 50 & 100 & 0 & 0 & 100 & 100 & 0 & 1,000 & 0.5 \\
\hline Repomucenus valenciennei & 900 & 200 & 50 & 0 & 100 & 550 & 0 & 0 & 650 & 200 & 0 & 2,650 & 1.4 \\
\hline
\end{tabular}


Table 2 Species composition of fishes collected by shrimp beam trawl inside of Jinju Bay, Korea, from December 2014 to November 2015 (unit: ind. $/ \mathrm{km}^{2}$ ) (Continued)

\begin{tabular}{|c|c|c|c|c|c|c|c|c|c|c|c|c|c|}
\hline Scientific name & Dec. & Jan. & Mar. & Apr. & May & Jun. & Jul. & Aug. & Sep. & Oct. & Nov. & Total & $\%$ \\
\hline Amblychaeturichthys hexanema & 550 & 100 & 50 & 0 & 0 & 0 & 0 & 0 & 0 & 50 & 0 & 750 & 0.4 \\
\hline Tridentiger trigonocephalus & 100 & 600 & 200 & 350 & 0 & 50 & 0 & 0 & 0 & 0 & 0 & 1,300 & 0.7 \\
\hline Acanthogobius flavimanus & 50 & 200 & 0 & 0 & 0 & 0 & 0 & 0 & 0 & 0 & 0 & 250 & 0.1 \\
\hline Cryptocentrus filifer & 0 & 50 & 0 & 50 & 50 & 100 & 400 & 0 & 500 & 350 & 50 & 1,550 & 0.8 \\
\hline Acentrogobius pflaumi & 100 & 0 & 100 & 100 & 50 & 0 & 50 & 0 & 750 & 100 & 50 & 1,300 & 0.7 \\
\hline Tridentiger nudicervicus & 0 & 0 & 0 & 0 & 550 & 650 & 100 & 0 & 0 & 0 & 0 & 1,300 & 0.7 \\
\hline Trichiurus japonicus & 0 & 0 & 0 & 0 & 0 & 0 & 0 & 100 & 0 & 0 & 0 & 100 & 0.1 \\
\hline Pleuronichthys cornutus & 0 & 0 & 3,800 & 2,900 & 1,850 & 1,500 & 1,250 & 200 & 100 & 0 & 0 & 11,599 & 6.2 \\
\hline Kareius bicoloratus & 0 & 100 & 0 & 0 & 50 & 50 & 50 & 0 & 0 & 0 & 0 & 250 & 0.1 \\
\hline Pseudopleuronectes yokohamae & 100 & 150 & 50 & 50 & 100 & 150 & 100 & 100 & 150 & 50 & 50 & 1,050 & 0.6 \\
\hline Clidoderma asperrimum & 0 & 0 & 0 & 50 & 0 & 100 & 0 & 0 & 0 & 0 & 0 & 150 & 0.1 \\
\hline Pseudaesopia japonica & 0 & 0 & 50 & 0 & 0 & 0 & 0 & 0 & 0 & 0 & 0 & 50 & + \\
\hline Cynoglossus robustus & 1,900 & 0 & 0 & 0 & 0 & 0 & 0 & 0 & 0 & 0 & 50 & 1,950 & 1.0 \\
\hline Cynoglossus semilaevis & 150 & 0 & 0 & 0 & 0 & 0 & 0 & 0 & 0 & 0 & 0 & 150 & 0.1 \\
\hline Cynoglossus abbreviatus & 0 & 100 & 50 & 0 & 50 & 0 & 100 & 0 & 0 & 0 & 0 & 300 & 0.2 \\
\hline Cynoglossus joyneri & 50 & 400 & 0 & 0 & 0 & 0 & 0 & 300 & 0 & 0 & 0 & 750 & 0.4 \\
\hline Cynoglossus interruptus & 0 & 0 & 0 & 50 & 0 & 50 & 300 & 0 & 150 & 0 & 0 & 550 & 0.3 \\
\hline Rudarius ercodes & 50 & 0 & 0 & 0 & 0 & 0 & 0 & 0 & 0 & 0 & 0 & 50 & + \\
\hline Stephanolepis cirrhifer & 0 & 0 & 0 & 0 & 0 & 0 & 0 & 0 & 0 & 0 & 50 & 50 & + \\
\hline Takifugu niphobles & 100 & 0 & 0 & 0 & 50 & 150 & 0 & 0 & 0 & 0 & 0 & 300 & 0.2 \\
\hline No. of species & 31 & 22 & 20 & 22 & 29 & 26 & 19 & 15 & 16 & 14 & 15 & 63 & \\
\hline Total & 14,299 & 6,649 & 10,899 & 31,098 & 21,248 & 8,299 & 14,249 & 67,845 & 4,800 & 5,500 & 3,700 & 188,585 & 100 \\
\hline
\end{tabular}

Jinju Bay $\left(6.1-24.2^{\circ} \mathrm{C}\right)$ than outside $\left(8.1-23.3^{\circ} \mathrm{C}\right)$. The difference in bottom water temperature between the stations was greatest in summer, and there was no significant difference in water temperature between stations in winter (Bonferroni's test, $P>0.05)$. Salinities were consistently lower at the station inside the bay than outside throughout the year, with the highest salinity occurring in April 2015 inside and the lowest during July 2015 outside (Fig. 2).

\section{Fish species composition}

A total of 534,657 individual fishes per square kilometre (ind. $/ \mathrm{km}^{2}$ ), comprising of 81 species and 47 families, were collected at both stations. Of these, 188,585 ind./ $\mathrm{km}^{2}$ belonging to 63 species and 40 families were collected inside the bay, while 346,072 ind. $/ \mathrm{km}^{2}$ from 65 species and 42 families were caught outside the bay (Table 1).

The number of species was similar between stations, but the number of individuals was considerably higher at the station outside than the one inside the bay. The most dominant species was Nuchequula nuchalis, which occurred at a frequency of $26 \%$ inside Jinju Bay and at $27 \%$ outside the bay. The next most dominant species inside the bay were Thryssa kammalensis (18\%) and Zoarces gillii (16\%), and Liparis tanakae (17\%) and T. kammalensis (9\%) outside the bay.

Forty species of young fishes were caught inside the bay, comprising approximately one third of the total number of individuals inside the bay. Forty-seven species of young fishes were caught outside the bay, comprising $52 \%$ of individuals caught, indicating that more various species grow outside of the bay. Juvenile $P$. cornutus, $Z$. gillii, and Hemitripterus villosus were commonly collected inside the bay from March to June, while at the station outside Jinju Bay, L. tanakae was commonly caught from March to May and Pennahia argentata from September to November (Tables 2 and 3).

\section{Seasonal variation in species composition}

While trends between seasons could not be empirically tested due to the lack of replication in seasonal data, monthly data binned into categories that matched particular seasons in Korea showed some interesting patterns. Difference between these binned data can be used to infer potential seasonal changes. The number of fish species varied from 14 to 31 between the four binned 
Table 3 Species composition of fishes collected by shrimp beam trawl outside of Jinju Bay, Korea, from December 2014 to November 2015 (unit: ind. $/ \mathrm{km}^{2}$ )

\begin{tabular}{|c|c|c|c|c|c|c|c|c|c|c|c|c|c|}
\hline Scientific name & Dec. & Jan. & Mar. & Apr. & May & Jun. & Jul. & Aug. & Sep. & Oct. & Nov. & Total & $\%$ \\
\hline Okamejei kenojei & 200 & 850 & 200 & 150 & 250 & 200 & 100 & 300 & 500 & 300 & 150 & 3,200 & 0.9 \\
\hline Muraenesox cinereus & 0 & 0 & 0 & 0 & 0 & 0 & 0 & 150 & 300 & 400 & 150 & 1,000 & 0.3 \\
\hline Conger myriaster & 400 & 250 & 600 & 350 & 100 & 250 & 200 & 0 & 350 & 200 & 250 & 2,950 & 0.9 \\
\hline Thryssa kammalensis & 0 & 0 & 0 & 1,650 & 0 & 0 & 0 & 11,599 & 600 & 10,299 & 7,149 & 31,297 & 9.0 \\
\hline Thryssa adelae & 0 & 0 & 800 & 0 & 0 & 0 & 0 & 0 & 0 & 0 & 0 & 800 & 0.2 \\
\hline Sardinella zunasi & 0 & 0 & 0 & 0 & 0 & 0 & 0 & 0 & 0 & 50 & 0 & 50 & + \\
\hline Konosirus punctatus & 50 & 0 & 50 & 0 & 0 & 0 & 0 & 0 & 0 & 0 & 0 & 100 & + \\
\hline Saurida microlepis & 0 & 0 & 0 & 0 & 0 & 0 & 0 & 0 & 50 & 100 & 150 & 300 & 0.1 \\
\hline Coelorinchus multispinulosus & 50 & 50 & 0 & 0 & 0 & 0 & 0 & 0 & 0 & 0 & 0 & 100 & + \\
\hline Lophius litulon & 0 & 0 & 0 & 0 & 50 & 50 & 0 & 0 & 0 & 0 & 0 & 100 & + \\
\hline Sebastiscus marmoratus & 50 & 0 & 0 & 0 & 0 & 0 & 0 & 0 & 0 & 0 & 0 & 50 & + \\
\hline Inimicus japonicus & 0 & 0 & 250 & 600 & 250 & 50 & 100 & 0 & 0 & 0 & 200 & 1,450 & 0.4 \\
\hline Minous monodactylus & 0 & 0 & 0 & 0 & 0 & 0 & 0 & 0 & 50 & 0 & 0 & 50 & + \\
\hline Hypodytes rubripinnis & 500 & 250 & 700 & 0 & 0 & 0 & 0 & 0 & 0 & 0 & 0 & 1,450 & 0.4 \\
\hline Chelidonichthys spinosus & 100 & 0 & 0 & 100 & 300 & 400 & 50 & 0 & 50 & 300 & 1,100 & 2,400 & 0.7 \\
\hline Platycephalus indicus & 500 & 200 & 100 & 450 & 250 & 250 & 200 & 150 & 100 & 100 & 100 & 2,400 & 0.7 \\
\hline Hexagrammos otakii & 0 & 50 & 0 & 0 & 0 & 0 & 0 & 0 & 0 & 0 & 0 & 50 & + \\
\hline Ricuzenius pinetorum & 6,999 & 3,000 & 1,900 & 200 & 0 & 0 & 0 & 0 & 0 & 0 & 0 & 12,099 & 3.5 \\
\hline Hemitripterus villosus & 0 & 50 & 1,650 & 150 & 0 & 0 & 0 & 0 & 0 & 0 & 0 & 1,850 & 0.5 \\
\hline Liparis tanakae & 100 & 250 & 50,746 & 6,949 & 400 & 0 & 0 & 0 & 0 & 0 & 0 & 58,445 & 16.9 \\
\hline Lateolabrax maculatus & 0 & 0 & 0 & 50 & 0 & 0 & 0 & 0 & 0 & 0 & 0 & 50 & + \\
\hline Malakichthys wakiyae & 0 & 0 & 0 & 50 & 0 & 0 & 0 & 0 & 0 & 0 & 0 & 50 & + \\
\hline Apogon lineatus & 0 & 0 & 0 & 0 & 1,000 & 3,150 & 450 & 0 & 0 & 1,150 & 1,050 & 6,799 & 2.0 \\
\hline Sillago japonica & 0 & 50 & 0 & 1,300 & 450 & 550 & 600 & 2,600 & 1,300 & 200 & 250 & 7,299 & 2.1 \\
\hline Decapterus maruadsi & 0 & 0 & 0 & 0 & 0 & 0 & 0 & 0 & 0 & 150 & 0 & 150 & + \\
\hline Trachurus japonicus & 0 & 0 & 0 & 0 & 0 & 0 & 0 & 0 & 0 & 300 & 150 & 450 & 0.1 \\
\hline Nuchequula nuchalis & 250 & 0 & 3,900 & 7,799 & 150 & 0 & 50 & 11,149 & 950 & 47,546 & 21,198 & 92,993 & 26.9 \\
\hline Hapalogenys mucronatus & 0 & 0 & 0 & 50 & 0 & 0 & 0 & 0 & 0 & 0 & 0 & 50 & + \\
\hline Johnius grypotus & 0 & 0 & 0 & 500 & 0 & 0 & 50 & 100 & 0 & 0 & 0 & 650 & 0.2 \\
\hline Pennahia argentata & 0 & 0 & 0 & 50 & 1,150 & 2,100 & 750 & 8,299 & 1,150 & 6,549 & 2,500 & 22,548 & 6.5 \\
\hline Larimichthys polyactis & 0 & 0 & 0 & 350 & 0 & 0 & 0 & 0 & 0 & 0 & 0 & 350 & 0.1 \\
\hline Upeneus japonicus & 0 & 0 & 0 & 0 & 0 & 0 & 0 & 0 & 0 & 0 & 50 & 50 & + \\
\hline Halichoeres poecilopterus & 0 & 50 & 0 & 0 & 0 & 0 & 0 & 0 & 0 & 0 & 0 & 50 & + \\
\hline Zoarces gillii & 450 & 550 & 50 & 9,549 & 3,300 & 2,650 & 50 & 0 & 0 & 0 & 0 & 16,599 & 4.8 \\
\hline Chirolophis wui & 0 & 50 & 0 & 0 & 0 & 0 & 0 & 0 & 50 & 0 & 0 & 100 & + \\
\hline Pholis nebulosa & 1,350 & 50 & 50 & 100 & 150 & 150 & 0 & 0 & 0 & 0 & 50 & 1,900 & 0.5 \\
\hline Pholis fangi & 50 & 150 & 500 & 2,700 & 1,050 & 2,000 & 50 & 0 & 0 & 0 & 100 & 6,599 & 1.9 \\
\hline Champsodon snyderi & 0 & 0 & 0 & 0 & 0 & 0 & 0 & 0 & 0 & 0 & 200 & 200 & 0.1 \\
\hline Parapercis sexfasciata & 1,450 & 450 & 150 & 100 & 250 & 150 & 0 & 0 & 100 & 0 & 50 & 2,700 & 0.8 \\
\hline Uranoscopus chinensis & 0 & 0 & 0 & 0 & 0 & 0 & 0 & 0 & 0 & 0 & 50 & 50 & + \\
\hline Xenocephalus elongatus & 0 & 0 & 0 & 0 & 0 & 0 & 0 & 0 & 0 & 800 & 150 & 950 & 0.3 \\
\hline Repomucenus valenciennei & 250 & 100 & 0 & 0 & 1,100 & 1,650 & 850 & 1,000 & 700 & 4,000 & 3,950 & 13,599 & 3.9 \\
\hline Amblychaeturichthys hexanema & 100 & 950 & 750 & 0 & 9,349 & 1,900 & 200 & 50 & 900 & 5,400 & 7,849 & 27,448 & 7.9 \\
\hline
\end{tabular}


Table 3 Species composition of fishes collected by shrimp beam trawl outside of Jinju Bay, Korea, from December 2014 to November 2015 (unit: ind. $/ \mathrm{km}^{2}$ ) (Continued)

\begin{tabular}{|c|c|c|c|c|c|c|c|c|c|c|c|c|c|}
\hline Scientific name & Dec. & Jan. & Mar. & Apr. & May & Jun. & Jul. & Aug. & Sep. & Oct. & Nov. & Total & $\%$ \\
\hline Tridentiger trigonocephalus & 50 & 100 & 0 & 0 & 0 & 0 & 0 & 0 & 0 & 0 & 0 & 150 & + \\
\hline Acanthogobius flavimanus & 300 & 50 & 100 & 0 & 0 & 0 & 0 & 0 & 0 & 0 & 0 & 450 & 0.1 \\
\hline Ctenotrypauchen microcephalus & 50 & 0 & 0 & 0 & 0 & 0 & 0 & 0 & 0 & 0 & 0 & 50 & + \\
\hline Chaeturichthys stigmatias & 0 & 0 & 0 & 150 & 0 & 0 & 0 & 0 & 0 & 0 & 0 & 150 & + \\
\hline Cryptocentrus filifer & 0 & 0 & 0 & 0 & 100 & 200 & 0 & 0 & 0 & 0 & 0 & 300 & 0.1 \\
\hline Acentrogobius pflaumi & 0 & 0 & 450 & 0 & 400 & 0 & 150 & 50 & 950 & 150 & 400 & 2,550 & 0.7 \\
\hline Trichiurus japonicus & 0 & 0 & 0 & 0 & 0 & 0 & 0 & 200 & 0 & 0 & 50 & 250 & 0.1 \\
\hline Psenopsis anomala & 0 & 0 & 0 & 0 & 0 & 0 & 0 & 0 & 0 & 150 & 0 & 150 & + \\
\hline Pseudorhombus pentophthalmus & 0 & 0 & 0 & 0 & 0 & 0 & 0 & 0 & 0 & 100 & 1,550 & 1,650 & 0.5 \\
\hline Pleuronichthys cornutus & 0 & 0 & 900 & 450 & 550 & 450 & 50 & 200 & 100 & 0 & 0 & 2,700 & 0.8 \\
\hline Kareius bicoloratus & 50 & 100 & 0 & 0 & 50 & 0 & 0 & 0 & 0 & 0 & 0 & 200 & 0.1 \\
\hline Pseudopleuronectes yokohamae & 100 & 500 & 250 & 50 & 300 & 100 & 50 & 0 & 200 & 0 & 0 & 1,550 & 0.4 \\
\hline Pseudaesopia japonica & 50 & 300 & 150 & 200 & 250 & 150 & 0 & 0 & 0 & 0 & 0 & 1,100 & 0.3 \\
\hline Zebrias fasciatus & 0 & 0 & 0 & 0 & 0 & 0 & 0 & 0 & 50 & 0 & 0 & 50 & + \\
\hline Cynoglossus robustus & 50 & 0 & 0 & 0 & 0 & 50 & 0 & 0 & 0 & 0 & 0 & 100 & + \\
\hline Cynoglossus semilaevis & 50 & 0 & 0 & 0 & 0 & 0 & 0 & 0 & 0 & 0 & 0 & 50 & + \\
\hline Cynoglossus abbreviatus & 0 & 1,750 & 100 & 150 & 500 & 700 & 0 & 0 & 0 & 100 & 0 & 3,300 & 1.0 \\
\hline Cynoglossus joyneri & 0 & 800 & 450 & 50 & 350 & 400 & 250 & 650 & 400 & 700 & 1,750 & 5,800 & 1.7 \\
\hline Cynoglossus interruptus & 0 & 0 & 0 & 0 & 400 & 1,250 & 150 & 150 & 1,450 & 100 & 0 & 3,500 & 1.0 \\
\hline Stephanolepis cirrhifer & 0 & 0 & 0 & 0 & 0 & 0 & 0 & 0 & 0 & 50 & 0 & 50 & + \\
\hline Takifugu niphobles & 150 & 0 & 0 & 0 & 0 & 0 & 0 & 0 & 0 & 0 & 0 & 150 & + \\
\hline Lagocephalus wheeleri & 0 & 0 & 0 & 0 & 0 & 0 & 0 & 0 & 0 & 50 & 0 & 50 & + \\
\hline No. of species & 26 & 25 & 23 & 27 & 26 & 23 & 19 & 15 & 21 & 25 & 26 & 65 & \\
\hline Total & 13,699 & 10,949 & 64,795 & 34,247 & 22,448 & 18,798 & 4,350 & 36,647 & 10,299 & 79,244 & 50,596 & 346,072 & 100 \\
\hline
\end{tabular}

+ less than $0.1 \%$

seasons, with the highest values recorded at both stations in winter (Fig. 3a). The mean number of species tended to be high during winter and spring at both stations and lowest during autumn (inside the bay) and summer (outside the bay). Fish abundance varied by temporally peaking in summer inside the bay and autumn outside the bay. Abundance was the lowest inside the bay in autumn and outside the bay in winter (Fig. 3b). Greater fish abundances corresponded with high occurrences of $N$. nuchalis and T. kammalensis during August (Tables 2 and 3). Diversity indices ranged from 0.98 to 2.63 inside the bay and 1.06 to 2.62 outside the bay. The highest diversities were recorded in winter inside the bay and summer outside the bay (Fig. 3c).

\section{Multivariate analyses of fish assemblages}

Cluster analysis of the 14 common species indicated that less than $1 \%$ of the total abundance of fishes were collected from inside the bay. Species were divided into five groups at a similarity level of $60 \%$ (Fig. 4a). Group A consisted of L. tanakae, Z. gillii, Ricuzenius pinetorum, Pholis fangi, Repomucenus valenciennei, and Pholis nebulosi, with a high emergence in spring and winter. Group B consisted of N. nuchalis, P. argentata, Sillago japonica, and Conger myriaster and were collected continuously during the survey period. P. cornutus and Inimicus japonicus collected in abundance in spring and summer formed Group C. T. kammalensis collected in summer comprised Group D, and Cynoglossus robustus collected in autumn comprised Group E (Fig. 4b).

Common fish species collected outside the bay were divided into four groups at a similarity level of $60 \%$ (Fig. 5a). Amblychaeturichthys hexanema, P. argentata, R. valenciennei, S. japonica, Apogon lineatus, Cynoglossus joyneri, and $C$. interruptuss were caught in high abundance in spring, summer, and autumn and clustered into Group A. L. tanakae, Z. gillii, P. fangi, and Cynoglossus abbreviatus collected in spring and winter formed Group B. Group C comprised of $N$. nuchalis and T. kammalensis that concentrated in spring and autumn. $R$. pinetorum collected in the winter formed Group D (Fig. 5b). 

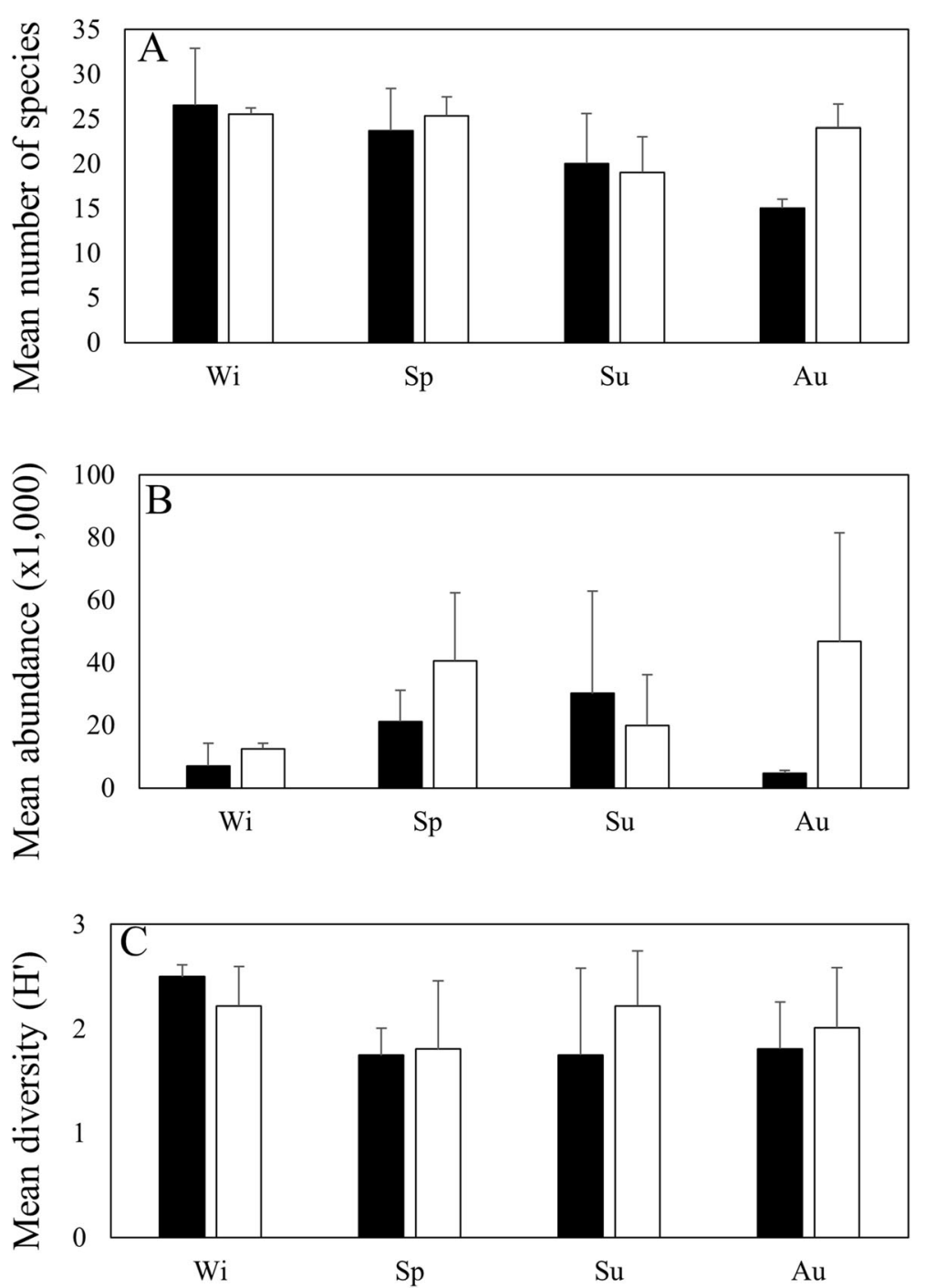

Fig. 3 Monthly variations in the number of species, number of individuals (ind./km²), and diversity index ( $H^{\prime}$ ) of fish collected inside (black) and outside (white) of Jinju Bay, Korea, from December 2014 to November 2015. Wi = winter, Sp = spring, Su = summer, Au = autumn

A canonical correspondence analysis revealed that three environmental factors contributed to fish assemblages between stations, and among common fish species in each of the four seasons (Fig. 6). Differences in fish assemblages between stations were linked to depth and salinity during winter, spring, and autumn, while temperature and depth contributed to differences in fish assemblages during summer (Fig. 6). Among the common fish species, $N$. nuchalis appeared negatively affected by salinity and depth in spring, summer, and autumn, but not in winter. P. cornutus appeared positively affected by water temperature in all seasons but autumn. T. kammalensis appeared positively affected by water temperature in summer and autumn, but was negatively affected by water temperature in spring.

\section{Spatio-temporal variations in common fish species}

In spring and autumn, the dominant species of fish at both stations were Z. gillii and N. nuchalis. However, the dominant species differed between stations in winter and summer. Pleuronichthys cornutus and N. nuchalis dominated inside the bay, while L. tanakae and T. kammalensis dominated outside the bay.

$N$. nuchalis occurred in low numbers at both stations in winter, but although the population increased rapidly inside the bay in the summer and outside the bay in autumn, there was no significant difference between the stations (ANOVA, $P=0.29$; Fig. 7a). T. kammalensis showed a rapidly increasing trend inside the bay in the summer and was collected in large numbers in both summer and autumn outside the bay (Fig. 7b). Z. gillii 


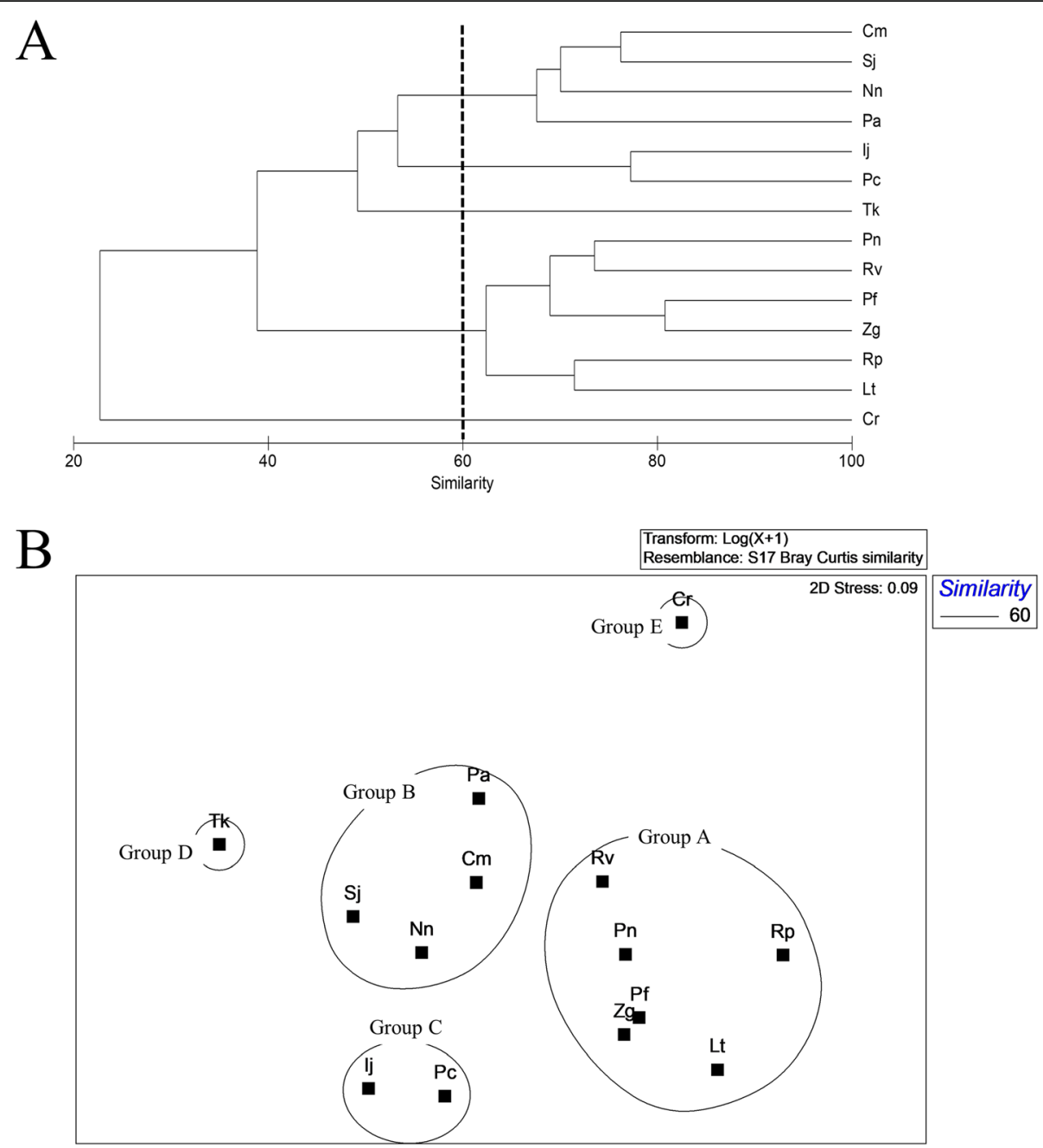

Fig. 4 A dendrogram (a) and non-metric multidimensional scaling (nMDS) (b) analysis of fish species collected by beam trawl inside of Jinju Bay, Korea (Nn, Nuchequula nuchalis; Tk, Thryssa kammalensis; Lt, Liparis tanakae; Zg, Zoarces gillii; Pa, Pennahia argentata; Rp, Ricuzenius pinetorum; Pf, Pholis fangi; Rv, Repomucenus valenciennei; Pc, Pleuronichthys cornutus; Sj, Sillago japonica; Cm, Conger myriaster; Cr, Cynoglossus robustus; Pn, Pholis nebulosa; lj, Inimicus japonicus)

and Pholis fangi were intensively collected inside and outside the bay in the spring (Fig. 7c, d). Pleuronichthys cornutus was collected in significantly higher numbers inside of the bay in spring compared to the other seasons (Bonferroni's test, $P<0.05$; Fig. 7e). L. tanakae was collected consistently outside the bay (ANOVA, $P=$ 0.34; Fig. 7f). Amblychaeturichthys hexanema was collected in abundance inside the bay in winter and outside the bay in spring and autumn (ANOVA, $P=0.20$; Fig. 7 g). Pennahia argentata was collected consistently outside the bay in summer and autumn (ANOVA, $P=0.14$; Fig. $7 \mathrm{~h})$.

\section{Size of juvenile fish}

Juvenile $P$. cornutus, $Z$. gillii, $H$. villosus, L. tanakae, and $P$. argentata were collected inside and outside the bay (Fig. 8). $P$. cornutus occurred during seven of the monthly surveys
(March-September) at both stations. These fish exhibited average lengths of $4.7 \mathrm{~cm} \pm 0.5 \mathrm{~cm}( \pm \mathrm{SD})$ in March and $17.3 \mathrm{~cm} \pm 1.1 \mathrm{~cm}$ in September. Z. gillii was collected over 3 months (April-June) inside the bay and over 4 months (April-July) outside the bay. H. villosus occurred over 3 months (March-May) inside the bay and for 2 months (March-April) outside the bay. These three species were collected more frequently from inside than from outside of the bay (Fig. 8). On the other hand, L. tanakae was found inside $(6.2-18.5 \mathrm{~cm} \mathrm{TL})$ and outside $(8.1-12.2 \mathrm{~cm} \mathrm{TL})$ of the bay over 3 months (March-May), but more individuals were collected at the station outside the bay than inside the bay. In addition, $P$. argentata (inside bay, $4.1-14.8 \mathrm{~cm}$ TL; outside of bay, $5.7-15.8 \mathrm{~cm}$ TL) were found more frequently outside the bay than inside, over 3 months (September-November). Xenocephalus elongatus occurred over 2 months (October-November), but only outside the bay. 

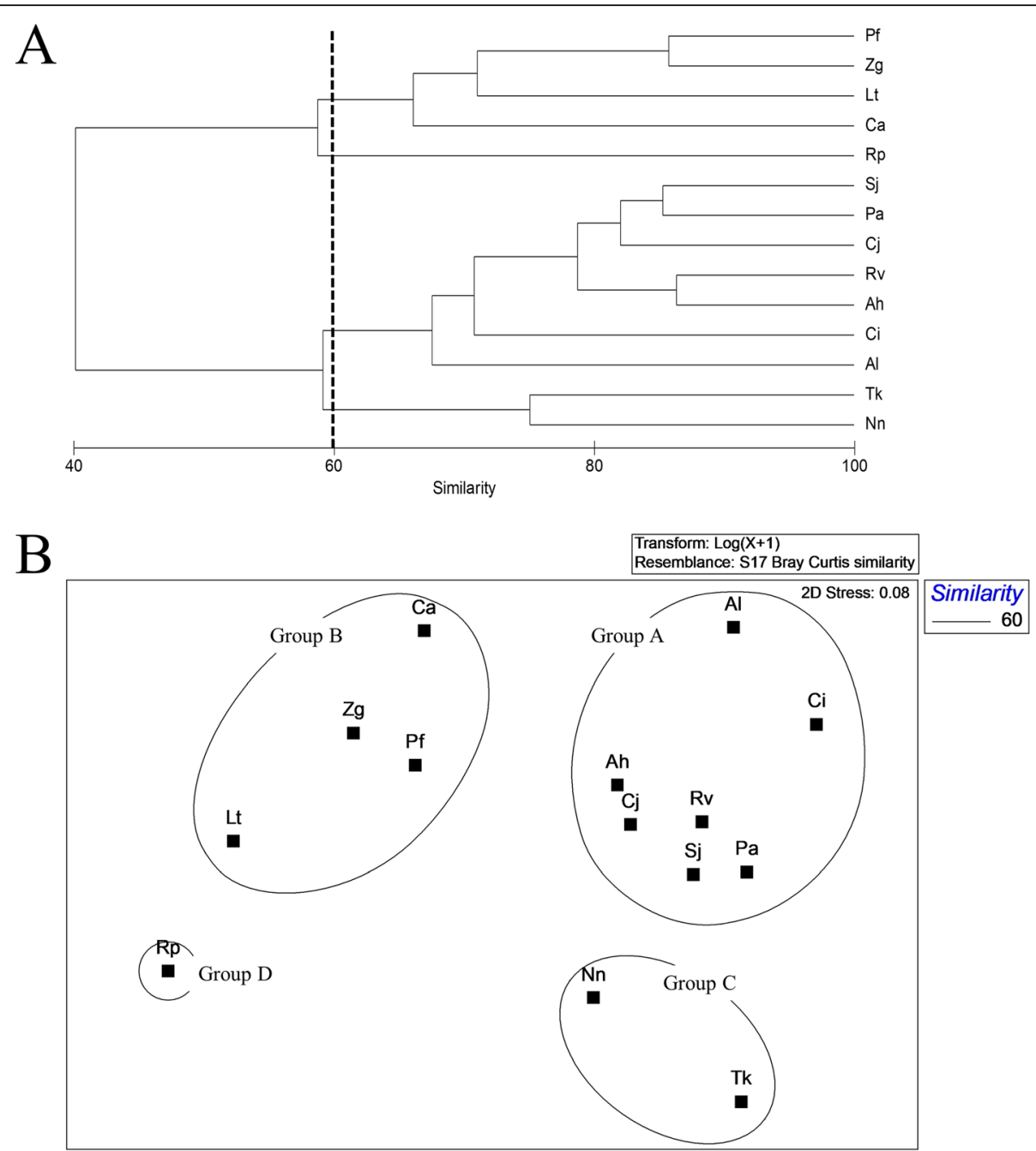

Fig. 5 A dendrogram (a) and non-metric multidimensional scaling (nMDS) (b) analysis of fish species collected by beam trawl outside of Jinju Bay, Korea (Nn, Nuchequula nuchalis; Tk, Thryssa kammalensis; Lt, Liparis tanakae; Zg, Zoarces gillii; Ah, Amblychaeturichthys hexanema; Pa, Pennahia argentata; Rp, Ricuzenius pinetorum; Pf, Pholis fangi; Rv, Repomucenus valenciennei; Sj, Sillago japonica; Al, Apogon lineatus; Cj, Cynoglossus joyneri; Ci, Cynoglossus interruptus; Ca, Cynoglossus abbreviatus)

All of these species were collected continuously throughout the study period, with TL increasing steadily over time, suggesting that these fish belonged to a single generation (Fig. 8).

\section{Discussion}

The consistent presence of juvenile fishes in the Jinju Bay region supports the concept that this broad area contains nursery grounds. The region appeared biodiverse in juvenile fishes, with 63 and 65 species collected inside and outside of the bay, respectively. Forty-seven of these species were collected simultaneously in both habitats. Of these, the most dominant species was $N$. nuchalis (26.4\%), followed by T. kammalensis (12.2\%), $L$. tanakae (12.0\%), Z. gillii (8.9\%), and A. hexanema (5.3\%). N. nuchalis is a semi-benthic fish that occurs near the substratum and tends to move in conspecific groups (Kim et al. 2005). Although Kim and Kang (1991) reported that $N$. nuchalis occurs in relatively low abundance in coastal waters around Shinsudo in the southern Korean sea, they have consistently been documented as a dominant fish species in coastal fish assemblages in southern Korea, including the Nakdong river estuary (Kwak and Huh 2003), Gwangyang bay (Kwak et al. 2012), and coastal waters of Gadeok Island (Jeong et al. 2013). In addition, young larvae of this species have also been recorded in the coastal ecosystems of Gwangyang Bay (Cha and Park 1994) and Nakdong River estuary (Choi et al. 2015).

The neritic region of the Korean Peninsula is documented to have a high level of primary productivity and has been proposed as a spawning ground, a nursery ground, 


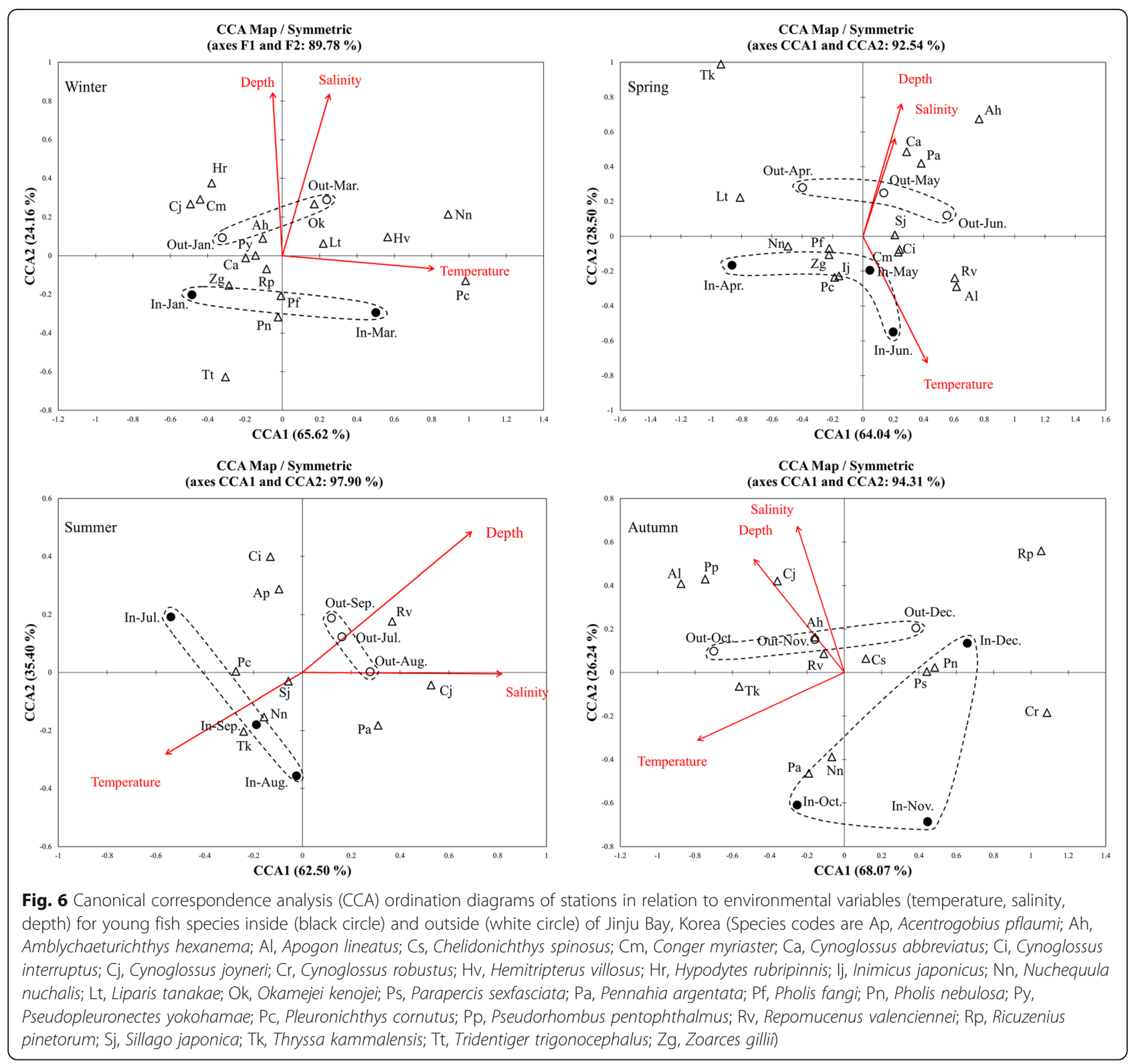

and a feeding site for many fish (Cha and Park, 1997). Song et al. (2019) collected young fish of L. tanakae, Z. gillii, C. joyneri, $P$. argentata, $P$. cornutus, and $P$. yokohamae around Jinju Bay and reported that they used Jinju Bay as a nursery ground and feeding site. In addition, most of the young fish individuals are collected in the estuary and bay, so it can be said to be a place for feeding site (Hwang et al. 2012). In addition, collected fish eggs and larvae to investigate fish that use Jinju Bay as a spawning and nursery ground. They found that 28 species use Jinju Bay as spawning grounds and 40 species use the area as a nursery ground. Most of the bays are rich in prey organisms under the influence of land, creating a favourable environment for young individuals (Newton et al. 2014; Álvarez et al. 2015).
In our study, $N$. nuchalis was the most dominant (inside the bay, 25.6\%; outside the bay, 26.9\%), followed by $T$. kammalensis $(17.9 \%$; 9.0\%) and Z. gillii $(16.0 \% ; 4.8 \%)$. All other species of juvenile fishes were caught in much lower abundances (Table 1). As such, the fish assemblages were dominated by a few fish species, and such dominance by minority species is a common phenomenon in most of estuarine habitats worldwide (e.g. Maes et al. 2005; Elliott et al. 2007; Selleslagh and Amara 2008). N. nuchalis is commonly seen as a dominant species in many estuarine habitats in Korea (Kwak and Huh 2003; Yoon et al. 2011; Jeong et al. 2013). It is also a species known to have the capacity to inhabit polluted waters (Lee 1996; Lee et al. 2011; Jeong et al. 2013). It may, therefore, be indicative of pollution in the waters around Jinju Bay. Future research 

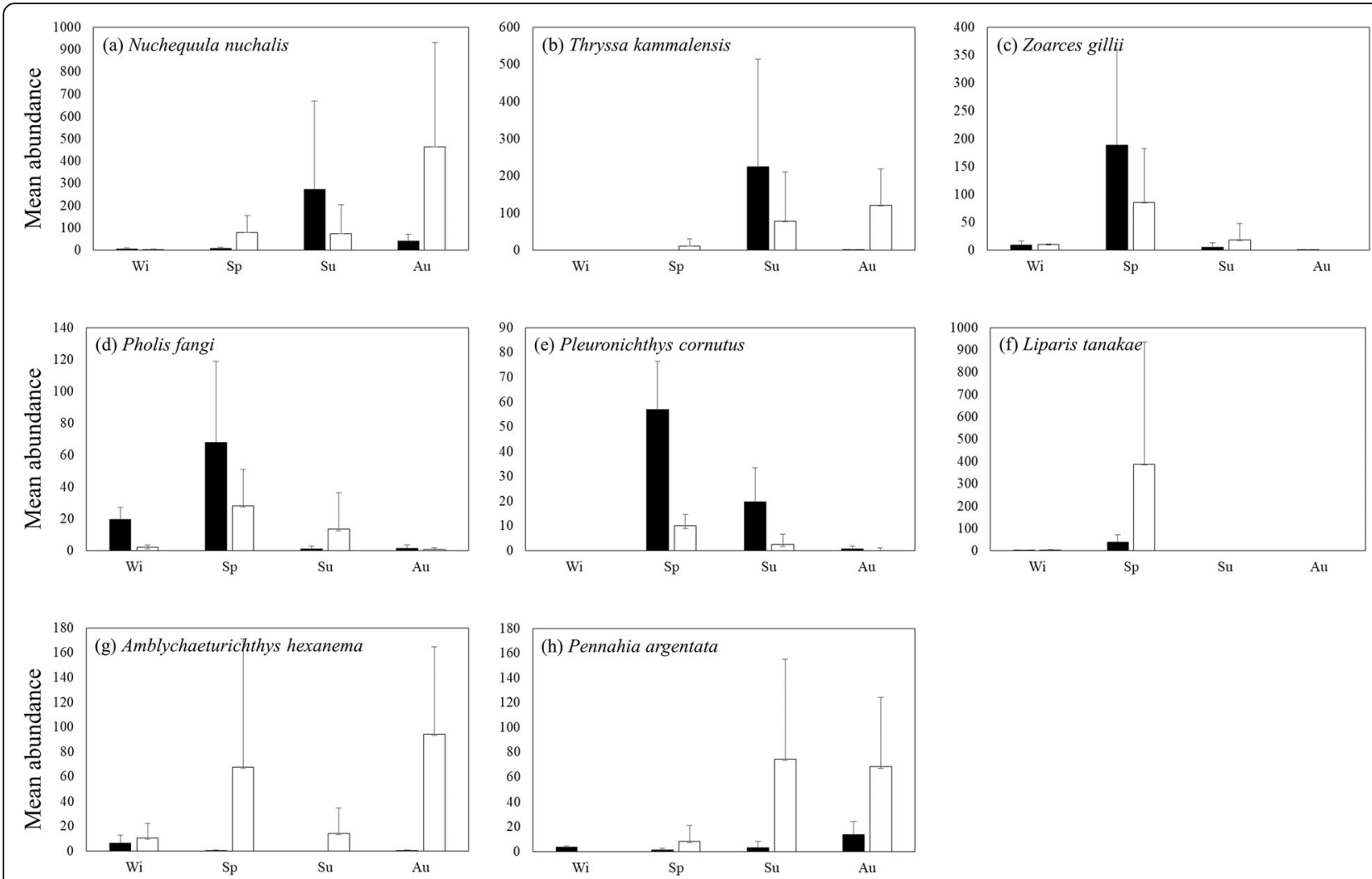

Fig. 7 Seasonal variations in mean abundance of 8 common fish species (a-h) between inside (black stick) and outside (white stick) of Jinju Bay, Korea, from December 2014 to November 2015

needs to be done to assess the relationship between pollution levels in Jinju Bay and the presence of N. nuchalis.

Sixteen species were collected within Jinju Bay only. Tridentiger nudicervicus (38.8\%) and Pseudoblennius cottoides (10.4\%) dominated the assemblage at this station whereas 18 species including Pseudorhombus pentophthalmus (40.2\%) and X. elongatus (23.2\%) dominated the assemblage outside the bay. These results revealed different fish assemblages inside and outside Jinju Bay, implying that different environment conditions, such as depth and salinity, may contribute towards optimizing habitat for each of the common species. During this study, salinities were lower inside Jinju Bay than at the station outside the bay. A substantial reduction in salinity was recorded in July within the bay that coincided with increased Nam River discharge derived from heavy rainfall at that time (Water Resources Management Information System WAMIS). This decrease in salinity has also been recorded at Gwangyang Bay (Kwak et al. 2012), Jinhae Bay (Hwang et al. 2011), and Masan Bay (Kwak and Park 2014). In addition, Nakdong River estuary (Park et al. 2015) located at the southern coast of Korea also indicated lowest salinity during the summer season. As with many rivers downstream in South Korea, Jinju Bay is an area that is heavily influenced by coastal water. According to a study by Chin et al. (2020), during the period of heavy rain in a similar estuary, salinity was reduced so substantially that anchovy spawning was stopped during this period. In addition, when there is substantial rain, photosynthesis of phytoplankton increases due to a decrease in salinity and an increase in organic matter (Park et al. 2013). After that, many phytoplankton die due to an anaerobic layer that forms on the surface of the water (Moon et al. 2006). The anoxic layer of the surface layer is also thought to have a substantial impact on pelagic eggs and larvae. During high rainfall, such as that experienced in Korea in July, the saline concentration decreases, thereby affecting the appearance or distribution of fish. Depth also differed between the stations, and this parameter may also influence the demography of species caught in this study (Muhling et al. 2007; Zhang et al. 2015). More research is required to understand the drivers behind the patterns observed in our research.

Estuaries provide spawning and nursery grounds for diversity of coastal fishes and estuarine residents (Hwang et al. 2005; Hwang and Rhow 2010; Lee et al. 2014; Park et al. 2015). In particular, juvenile $Z$. gillii, $P$. cornutus, $L$. tanakae, $H$. villosus, $P$. argentata, and $X$. elongates were collected continuously throughout the year, indicating the probability of fish nurseries inside and outside Jinju 

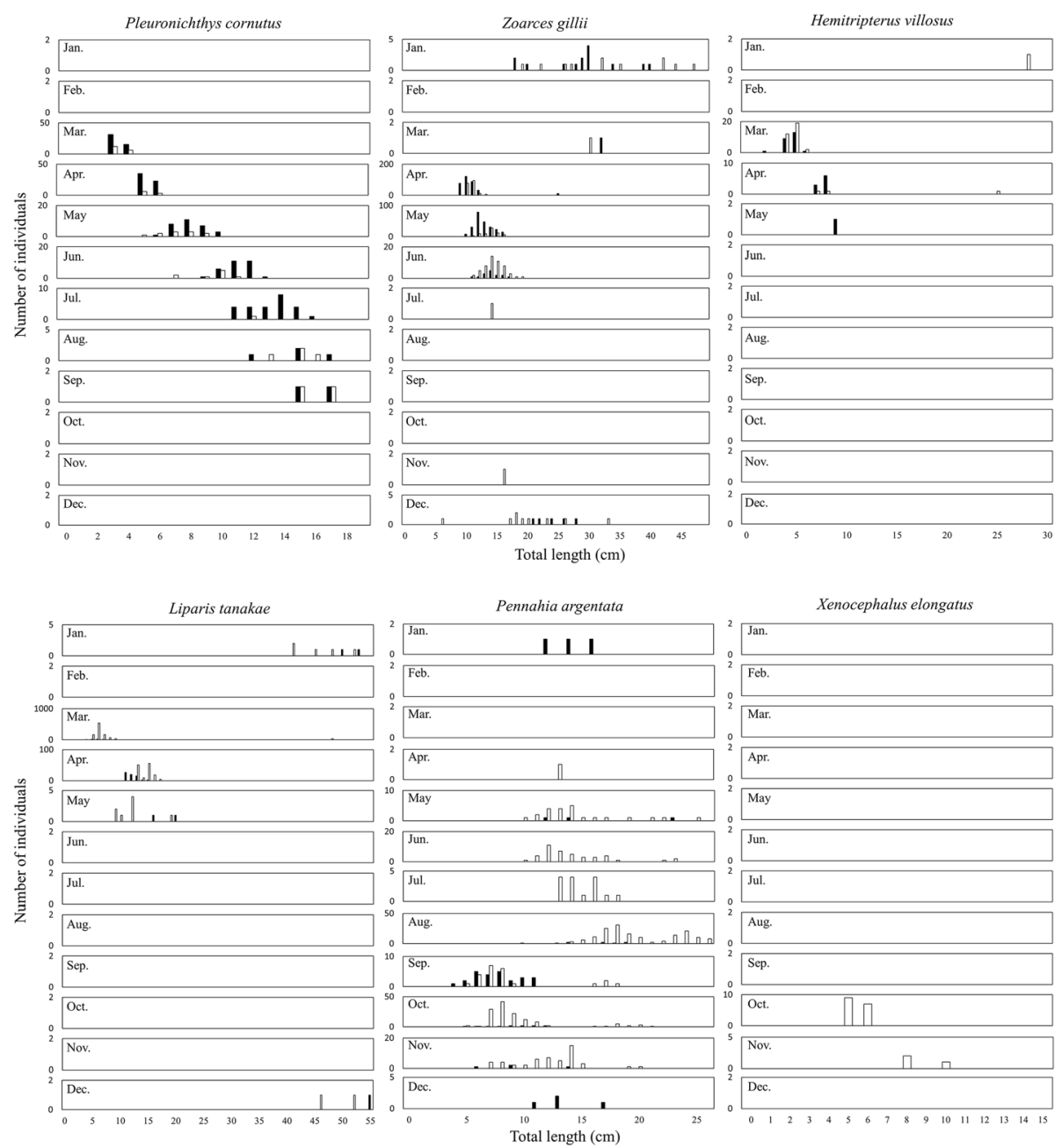

Fig. 8 Monthly change in length-frequency distribution of the six species caught with a beam trawl inside (black stick) and outside (white stick) of Jinju Bay, Korea, from December 2014 to November 2015

Bay due to the continued residency of these species. The location of the proposed nursery grounds differed depending on the species of fish. Z. gillii, P. cornutus, and H. villosus were resident species at the station inside Jinju Bay and are likely to use this areas as a nursery ground, whereas $L$. tanakae, $P$. argentata, and $X$. elongates were resident at the station outside the bay and potentially used this area as a separate nursery ground. In addition, the timing of residency differed from between species. Such difference temporal and spatial differences in occurrence may be a reflection of the spawning patterns of these species or may occur as avoidance of competition for food and habitat, as observed in other juvenile fish (Amara et al. 2001). If suchareas are acting as nursery grounds, food provisioning and refuge from predation for these juvenile fishes should be higher in these areas than in other regions (McLusky and Elliott 2004).

It is well known that offshore marine aquatic resources in the region are depleted due to overfishing and environmental pollution (Yoo et al. 1999; Zhang et al. 2003). For the conservation and management of aquatic biological resources in the region, various management regimes have been implemented, such as establishing a catch prohibition length and period (Cha and Jung 2012; Ji et al. 2015). Peterson et al. (2004) said that protecting spawning grounds during the spawning season is the most effective way to conserve such resources. Protection of the two proposed nursery grounds identified in this study should also be considered during spawning times of the relevant species.

Our research identified potential nursery grounds in Jinju Bay from collecting juvenile fishes over time. However, such functions are also verified throughout various methods, including analyses of single-nucleotide polymorphisms (SNPs) and trace elements (e.g. Sr, Ba, Cr) composition in fish body, because those methods have broadly been applied for estimating the sea areas of spawning grounds and migration routes during early life history 
(Rooker et al. 2008; Nielsen et al. 2012; Bonanomi et al. 2016; Shiao et al. 2016). Thus, further studies are recommended via analysing trace elements of otoliths in both inside and outside Jinju Bay, and/or single-base polymorphism.

\section{Conclusions}

Jinju Bay is a semi-closed bay whose salinity is affected by freshwater discharge from the Nam River, particularly in summer. Nevertheless, 81 species were collected from inside and outside of Jinju Bay, and it was found that various fish were inhabited. Also, 40 species used inside of the bay as a nursery ground. Since outside of the bay has less environmental change than inside of the bay, more species ( 47 species) used inside of the bay as nursery ground. Especially Pleuronichthys cornutus, Zoarces gillii, and Hemitripterus villosus used inside of the bay as nursery ground. Meanwhile, Liparis tanakae, Pennahia argentata, and Xenocephalus elongates used outside of the bay.

\section{Acknowledgements}

We thank Joo Myun Park (KIOST) for help with this paper. Also, we would like to deeply thank all members of Ichthyological laboratory in Pukyong National University for help in our sampling.

\section{Authors' contributions}

SHM and JKK contributed to the conceptualization and design of research. SHM, SNK, WCL, JBK, HCK, and JKK contributed to the investigations and experiments. SHM and JKK contributed to the data analysis. SHM and JKK contributed to the writing of the original draft. SHM, JEW, and JKK contributed to the writing including review and editing. The author(s) read and approved the final manuscript.

\section{Funding}

This research was supported by the Marine Biotechnology Program of the Korea Institute of Marine Science and Technology Promotion (KIMST) funded by the Ministry of Oceans and Fisheries (MOF) (No. 20170431), and the National Institute of Fisheries Science (R2020048).

\section{Availability of data and materials}

Not applicable.

\section{Ethics approval and consent to participate}

Not applicable

\section{Consent for publication}

Not applicable

\section{Competing interests}

The authors declare that they have no competing interests.

\section{Author details}

${ }^{1}$ Department of Biological Sciences, Macquarie University, North Ryde, NSW 2109, Australia. Environ-Ecological Engineering Institute Co., Ltd., 97, Centum jungang-ro, Haeundae-gu, Busan, South Korea. ${ }^{3}$ Department of Marine Biology, Pukyong National University, Busan 48513, South Korea. ${ }^{4}$ National Institute of Fisheries Science, Busan 46083, South Korea.

Received: 30 June 2020 Accepted: 6 November 2020

Published online: 18 December 2020

\section{References}

Able KW, Fahay MP. Ecology of estuarine fishes: temperate waters of the western North Atlantic. Baltimore: Johns Hopkins University Press; 2010.
Allen LG. Seasonal abundance, composition, and productivity of the littoral zone assemblage in upper Newport Bay, California. Fish Bull. 1982;80:769-90.

Álvarez I, Catalán IA, Jordi A, Alemany F, Basterretxea G. Interaction between spawning habitat and coastally steered circulation regulate larval fish retention in a large shallow temperate bay. Estuar Coast Shelf Sci. 2015;167: 377-89.

Amara R, Laffargue P, Dewarumez J, Maryniak C, Lagardère F, Luzac C. Feeding ecology and growth of O-group flatfish (sole, dab and plaice) on a nursery ground (Southern Bight of the North Sea). J Fish Biol. 2001;58:788-803.

Bonanomi S, Overgaard Therkildsen N, Retzel A, Berg Hedeholm R, Wæver Pedersen M, Meldrup D, Pampoulie C, Hemmer-Hansen J, Nkjær PG, Nielsen EE. Historical DNA documents long distance natal homing in marine fish. Mol Ecol. 2016;25:2727-34.

Bray JR, Curtis JT. An ordination of the upland forest communities of southern Wisconsin. Ecol monogr. 1957;27:325-49.

Cha HK, Jung S. Simulation-based yield-per-recruit analysis of Pacific cod Gadus macrocephalus in southeastern Korean coastal waters. Korean J Fish Aquat Sci. 2012;45:493-8.

Cha SS, Park KJ. Distribution of the ichthyoplankton in Kwangyang Bay. Korean J Ichthyol. 1994;6:60-70.

Cha SS, Park KJ. Seasonal changes in species composition of fishes collected with a bottom trawl in Kwangyang bay, Korea. Korean J Ichthyol. 1997;9:235-43.

Chin BS, Kim ST, Kim JS, Park GS. Species composition and abundances of ichthyol-plankton in Geum River Estuary in Spring and Summer. J Kor Soc Fish Mar Edu. 2020;32:65-73.

Choi HC, Park JM, Huh SH. Spatio-temporal variations in species composition and abundance of larval fish assemblages in the Nakdong River estuary, Korea. Korean J Ichthyol. 2015:27:104-15.

Clarke KR, Gorley RN. PRIMER v6.1.6. User Manual/Tutorial. Plymouth: PRIMER-E, Plymouth Marine Laboratory; 2006.

Elliott M, Dewailly F. The structure and components of European estuarine fish assemblages. Aquat Ecol. 1995:29:397-417.

Elliott M, Whitfield AK, Potter IC, Blaber SJ, Cyrus DP, Nordlie FG, Harrison TD. The guild approach to categorizing estuarine fish assemblages: a global review. Fish Fish. 2007:8:241-68.

Faria A, Morais P, Chícharo MA. Ichthyoplankton dynamics in the Guadiana estuary and adjacent coastal area, South-East Portugal. Estuar Coast Shelf Sci. 2006:70:85-97.

Grol MG, Nagelkerken I, Rypel AL, Layman CA. Simple ecological trade-offs give rise to emergent cross-ecosystem distributions of a coral reef fish. Oecologia. 2011;165:79-88.

Hwang OM, Shin KS, Baek SH, Lee WJ, Kim SA, Jang MC. Annual variations in community structure of mesozooplankton by short-term sampling in Jangmok Harbor of Jinhae Bay. Ocean Polar Res. 2011;33:235-53.

Hwang SD, Lee WJ, Im YJ. Comparison of nekton assemblage structures between estuary and inshore waters on the mid-western coast of Korea. The Sea. 2012;17:149-59.

Hwang SD, Rhow JG. Seasonal variation in species composition of estuarine fauna collected by a stow net in the Han River estuary on the mid-western coast of Korea. The Sea. 2010;15:72-85.

Hwang SW, Hwang HB, Noh HS, Lee TW. Seasonal variation in species composition of fish collected by a bag net in the Geum River estuary, Korea. Korean J Fish Aquat Sci. 2005;38:39-54

Jeong JM, Park JM, Huh SH, Ye SJ, Kim HJ, Baeck GW. Seasonal variation in the species composition of the fish assemblages in the coastal waters off Gadeok-do, south sea, Korea. Korean J Fish Aquat Sci. 2013:46:948-56

Ji HS, Lee DW, Choi JH, Choi KH. Development of naturally-spawned Pacific herring Clupea pallasii larvae. Korean J Fish Aquat Sci. 2015;48:362-7.

Jung KY, Ro YJ. Stratification and destratification processes in the Kangjin Bay, South Sea, Korea. The Sea. 2010;15:97-109.

Kang CK, Baik MS, Kim JB, Lee PY. Seasonal and spatial distribution of soft-bottom Polychaetesin Jinju Bay of the Southern Coast of Korea. Korean J Fish Aquat Sci. 2002;35:35-45.

Kang YS, Chae YK, Lee HR. Variation of density stratification due to fresh water discharge in the Kwangyang Bay and Jinju Bay. J Korean Soc Coast Ocean Eng. 2011;23:126-37.

Kim CK, Kang YJ. Fish assemblage collected by gill net in the coastal shallow water off Shinsudo, Samchonpo. Korean J Fish Aquat Sci. 1991;24:99-110.

Kim CK, Lee JT, Jang HS. Water circulation structure in the Chinju Bay of Korea. J Korean Soc Coast Ocean Eng. 2010;22:215-23. 
Kim IS, Choi Y, Lee CL, Lee YJ, Kim BJ, Kim JH. Illustrated book of Korean fishes, vol. 615. Seoul: Kyo-Hak Publishing Co; 2005.

Kim JK, Ryu JH. Distribution map of sea fishes in Korea, vol. 667. Busan: Maple publishing Co; 2016.

Kurita Y, Uehara S, Okazaki Y, Sakami T, Nambu R, Tomiyama T. Impact of the great tsunami in 2011 on the quality of nursery grounds for juvenile Japanese flounder Paralichthys olivaceus in Sendai Bay, Japan. Fish Oceanogr. 2017;26:165-80

Kwak SN, Huh S. Changes in species composition of fishes in the Nakdong River estuary. Korean J Fish Aquat Sci. 2003;36:129-35.

Kwak SN, Huh SH, Kim HW. Change in fish assemblage inhabiting around Dae Island in Gwangyang Bay, Korea. Korean Soc Mar Environ Saf. 2012; 18:175-84.

Kwak SN, Park JM. Temporal and spatial variation in species composition and abundances of ichthyoplankton in Masan Bay. Korean J Ichthyol. 2014;26:42-9.

Lee SK, Choi MS, Seo YI, Lee JB. Seasonal species composition and cluster analysis of catches by shrimp beam trawl in the Geum river estuary. J Korean soc Fish Ocean Technol. 2014;50:455-66.

Lee SK, Seo Yl, Kim Jl, Kim HY, Choi MS. Seasonal species composition and fluctuation of fishes by beam trawl in Yeoja Bay. Korean J Ichthyol. 2011;23:206-16.

Lee TW. Change in species composition of fish in Chonsu Bay 1. Demersal fish. Korean J Fish Aquat Sci. 1996;29:71-83.

Lin HY, Chiu MY, Shih YM, Chen IS, Lee MA, Shao KT. Species composition and assemblages of ichthyoplankton during summer in the East China Sea. Contin Shelf Res. 2016:126:64-78.

Maes J, Stevens M, Ollevier F. The composition and community structure of the ichthyofauna of the upper Scheldt estuary: synthesis of a 10-year data collection (1991-2001). J Appl Ichthyol. 2005;21:86-93.

McLusky DS, Elliott M. The estuarine ecosystem: ecology, threats and management, third ed. Oxford: Oxford University Pres. 2004;214.

Moon SY, Soh HY, Choi SD, Jung CS, Kim SY, Lee YS. Effect of a low-oxygen layer on the vertical distribution of zooplankton in Gamak Bay. Korean J Environ Biol. 2006;24:240-7.

Muhling B, Beckley LE, Olivar MP. Ichthyoplankton assemblage structure in two meso-scale Leeuwin Current eddies, eastern Indian Ocean. Deep Sea Res Part 2 Top Stud Oceanogr. 2007;54:1113-28.

Nakabo T. Fishes of Japan with pictorial keys to the species. Hadano: Tokai University Press; 2013. I: xlix+ 1-864; II: xxxii+ 865-1747; III: xvi+ 1751-2428.

Nelson JS, Grande TC, Wilson MV. Fishes of the World. 5th ed. Hoboken: Wiley; 2016.

Newton A, Icely J, Cristina S, Brito A, Cardoso AC, Colijn F, Dalla-Riva S, Gertz F, Hansen J, Holmer M, Ivanova K, Leppäkoski E, Mocenni C, Mudge S, Murray N, Pejrup M, Razinkovas A, Reizopoulou S, Pérez-Ruzafa A, Schernewski G, Schubert H, Seeram L, Solidoro C, Viaroli P, Zaldívar JM. An overview of ecological status, vulnerability and future perspectives of European large shallow, semi-enclosed coastal systems, lagoons and transitional waters. Estuar Coast Shelf Sci. 2014;140:95-122.

Nielsen EE, Cariani A, Mac Aoidh E, Maes GE, Milano I, Ogden R, Taylor M, Hemmer-Hansen J, Babbucci M, Bargelloni L, Bekkevold D, Diopere E, Grenfell L, Helyar S, Limborg MT, Martinsohn JT, McEwing R, Panitz F, Patarnello T, Tinti F, Van Houdt JKJ, Volckaert FAM, Waples RS, Consortium F, Carvalho GR. Gene-associated markers provide tools for tackling illegal fishing and false eco-certification. Nat Commun. 2012;3:1-7.

Oh HJ, Lee YH, Yang JH, Kim SH. The characteristics of phytoplankton distributions related to the oceanographic conditions in the Southern Waters of the Korean in summer, 2004. J Kor Associ Geogra Info Stud. 2007:10:40-8.

Park BI. A study on the main paths of water vapor transportation in cases of the heavy rainfall of the south coastal region of Korea for Changma season. J Geog. 2005;49:175-85.

Park JG, Choi CH, Jung SW, Yun SM, Kim SH. Changes in phytoplankton communities and environmental factors in Saemangeum artificial lake, South Korea between 2006 and 2009. Korean J Environ Biol. 2013;31:213-24.

Park JM, Huh SH, Baeck GW. Temporal variations of fish assemblage in the surf zone of the Nakdong River Estuary, southeastern Korea. Anim Cells Syst. 2015:19:350-8

Peterson M, Comyns B, Rakocinski C. Defining the fundamental physiological niche of young estuarine fishes and its relationship to understanding distribution, vital metrics, and optimal nursery conditions. Environ Biol Fishes. 2004;71:143-9.

Rooker JR, Secor DH, De Metrio G, Schloesser R, Block BA, Neilson JD. Natal homing and connectivity in Atlantic bluefin tuna populations. Science. 2008; 322:742-4.
Selleslagh J, Amara R. Environmental factors structuring fish composition and assemblages in a small macrotidal estuary (eastern English Channel). Estuar Coast Shelf Sci. 2008;79:507-17.

Selleslagh J, Amara R, Laffargue P, Lesourd S, Lepage M, Girardin M. Fish composition and assemblage structure in three Eastern English Channel macrotidal estuaries: a comparison with other French estuaries. Estuar Coast Shelf Sci. 2009;81:149-59.

Shannon CE, Weaver W. The mathematical theory of communication, vol. 117. Urbana: Illinois Univ Press; 1949.

Shiao JC, Chen CY, Zhang J, lizuka Y. Habitat use and migratory life history of Salangid icefish (Salangidae) revealed by otolith Sr/Ca ratios. Zool Stud. 2016;55:e3.

Song MY, Kim JI, Kim ST, Lee JH, Lee JB. Seasonal variation in species composition of catch by a coastal beam trawl in Jinhae Bay and Jinju Bay, Korea. J Korean Soc Fish Ocean Technol. 2012;48:428-44.

Song SH, Jeong JM, Lee SH, Kim DH. Species composition and community structure of fish by shrimp beam trawl between Sacheon Bay and coastal waters off Namhae, Korea. J Korean Soc Fish Ocean Technol. 2019;55:217-32.

Swearer SE, Caselle JE, Lea DW, Warner RR. Larval retention and recruitment in an island population of a coral-reef fish. Nature. 1999:402:799-802.

Vasconcelos RP, Reis-Santos P, Maia A, Fonseca V, França S, Wouters N, Costa MJ, Cabral HN. Nursery use patterns of commercially important marine fish species in estuarine systems along the Portuguese coast. Estuar Coast Shelf Sci. 2010;86:613-24.

Yamane K, Murase I, Shirafuji N, Hayashi A, Nagakura Y, Watanabe Y. Nursery habitat use for larval and juvenile Pacific herring Clupea pallasii in Miyako Bay on the Pacific coast of northern Japan. Fish Sci. 2019:85:407-16.

Yeo HG, Park MO. Seasonal variations of phytoplankton community and water quality in the east area of Chinhae bay. J Environ Sci Int. 1997;6:231-8.

Yoo JM, Lee EK, Kim S. Distribution of ichthyoplankton in the adjacent waters of Yousu. Korean J Fish Aquat Sci. 1999;32:295-302.

Yoon HS, An YK, Choi SD. Species composition and seasonal variation of fish assemblages in Sargassum beds in Gamak Bay, Korea. J Korean Soc Mar Environ Saf. 2011;17:15-21.

Zhang $\mathrm{Cl}$, Lee SI, Kim JM. Ecosystem-based management of fisheries resources in marine ranching areas. J Korean Soc Fish Res. 2003;6:71-83.

Zhang H, Xian W, Liu S. Ichthyoplankton assemblage structure of springs in the Yangtze Estuary revealed by biological and environmental visions. PeerJ. 2015:3:e1186.

\section{Publisher's Note}

Springer Nature remains neutral with regard to jurisdictional claims in published maps and institutional affiliations.

\section{Ready to submit your research? Choose BMC and benefit from:}

- fast, convenient online submission

- thorough peer review by experienced researchers in your field

- rapid publication on acceptance

- support for research data, including large and complex data types

- gold Open Access which fosters wider collaboration and increased citations

- maximum visibility for your research: over $100 \mathrm{M}$ website views per year

At BMC, research is always in progress.

Learn more biomedcentral.com/submission 